\title{
Quantum entanglement and classical communication through a depolarising channel
}

\author{
Dagmar Bruß, \\ Institut für Theoretische Physik, Universität Hannover, Appelstr. 2, D-30167 Hannover, Germany \\ Lara Faoro, \\ Dipartimento di Fisica, Politecnico di Torino, Via Duca degli Abruzzi 24, I-10129 Torino, Italy \\ Chiara Macchiavello, \\ Dipartimento di Fisica "A.Volta" and INFM-Unità di Pavia, Via Bassi 6, I-27100 Pavia, Italy \\ G.Massimo Palma \\ Dipartimento di Scienze Fisiche ed Astronomiche and INFM-Unità di Palermo, Via Archirafi 36, I-90123 Palermo, Italy
}

August 11, 2018

\begin{abstract}
We analyse the role of entanglement for transmission of classical information through a memoryless depolarising channel. Using the isotropic character of this channel we prove analytically that the mutual information cannot be increased by encoding classical bits into entangled states of two qubits.
\end{abstract}

\section{Introduction}

Entanglement is probably the most important resource in quantum information processing: quantum teleportation [1], quantum cryptography [2], quantum computation [3], quantum error correction 近] are only some examples of its ubiquitous crucial role. In all the instances mentioned above its main use is transmitting, processing, correcting quantum information. In this paper we would like to analyse whether it is useful in one particular scenario of transmission of classical information along a noisy channel: the depolarising channel. It has been proved that when multiple uses of the channel are allowed entangled states can maximise distinguishability in a particular case of noisy channel: the two-Pauli channel [5]. It is however common belief that, due to their fragility in the presence of noise, entangled states cannot improve the transmission of classical information when used as signal states, at least when the noise is isotropic - in the sense that will be explained in the following sections - as in the case of the depolarising channel. Although there is numerical evidence in this direction [5] no explicit proof has been produced so far, at least at the best of the authors' knowledge. The scope of this paper is precisely to prove that the mutual information cannot be increased when signals of entangled states of two qubits are used with a memoryless depolarising channel.

\section{Description of the Channel}

In the following we will consider a memoryless quantum channel acting on individual qubits. The channel is described by "action" operators [6] $A_{k}$ satisfying $\sum_{k} A_{k}^{\dagger} A_{k}=\mathbb{1}$ such that if we send through the channel a qubit in a state described by the density operator $\pi$ the output qubit state is given by the map

$$
\pi \longrightarrow \Phi(\pi)=\sum_{k} A_{k} \pi A_{k}^{\dagger}
$$


By definition a channel is memoryless when its action on arbitrary signals $\pi_{i}$, consisting of $n$ qubits (including entangled ones), is given by

$$
\Phi\left(\pi_{i}\right)=\sum_{k_{1} \cdots k_{n}}\left(A_{k_{n}} \otimes \cdots \otimes A_{k_{1}}\right) \pi_{i}\left(A_{k_{1}}^{\dagger} \otimes \cdots \otimes A_{k_{n}}^{\dagger}\right)
$$

For the depolarising channel, on which we will concentrate our attention, the action operators take the following simple form

$$
A_{0}=\frac{1}{2} \sqrt{1+3 \eta} \mathbb{1}, \quad A_{x, y, z}=\frac{1}{2} \sqrt{1-\eta} \sigma_{x, y, z} .
$$

Here $\sigma_{x, y, z}$ are the Pauli matrices, which are transformed under the action of the channel as

$$
\sum_{k} A_{k} \sigma_{l} A_{k}^{\dagger}=\eta \sigma_{l}
$$

As we can see, the depolarising channel can be specified by the parameter $\eta$, whose meaning will be clear in the following section, or equivalently by the error probability $p_{e}=3(1-\eta) / 4$. This gives us a complete description of the channel for any input state composed of an arbitrary number of qubits.

\section{Entanglement and mutual information}

In the simplest scenario the transmitter can send one qubit at a time along the channel. In this case the codewords will be restricted to be the tensor product of the states of the individual qubits. Quantum mechanics however allows also the possibility to entangle multiple uses of the channel. For this more general strategy it has been shown that the amount of reliable information which can be transmitted per use of the channel is given by $[7,8$

$$
C_{n}=\frac{1}{n} \sup _{\mathcal{E}} I_{n}(\mathcal{E})
$$

where $\mathcal{E}=\left\{p_{i}, \pi_{i}\right\}$ with $p_{i} \geq 0, \sum p_{i}=1$ is the input ensemble of states $\pi_{i}$ of $n$ - generally entangled qubits and $I_{n}(\mathcal{E})$ is the mutual information

$$
I_{n}(\mathcal{E})=S(\rho)-\sum_{i} p_{i} S\left(\rho_{i}\right)
$$

where the index $n$ stands for the number of uses of the channel. Here

$$
S(\chi)=-\operatorname{tr}(\chi \log \chi)
$$

is the von Neumann entropy, $\rho_{i}=\Phi\left(\pi_{i}\right)$ are the density matrices of the outputs and $\rho=\sum_{i} p_{i} \rho_{i}$. Logarithms are taken to base 2. The advantage of the expression (6) is that it includes an optimisation over all possible POVMs at the output, including collective ones. Therefore no explicit maximisation procedure for the decoding at the output of the channel is needed.

The interest for the possibility of using entangled states as channel input is that it cannot generally be excluded that $I_{n}(\mathcal{E})$ is superadditive for entangled inputs, i.e. we might have $I_{n+m}>I_{n}+I_{m}$ and therefore $C_{n}>C_{1}$.

In this scenario the classical capacity $C$ of the channel is defined as

$$
C=\lim _{n \rightarrow \infty} C_{n} .
$$

For the depolarising channel a lower bound on $C$ is given by the one-shot capacity $C_{1}$ (see [7]), while upper bounds are given in [9]. In this paper we will not attempt to find the classical capacity of the depolarising channel, as this would imply analysing signals with any degree of entanglement between $n$ qubits and 
performing the limit $n \rightarrow \infty$. We will restrict ourselves to the simplest non-trivial case, namely $n=2$, and we will find the maximal mutual information $I_{2}(\mathcal{E})$.

The question we want to address is: is it possible to increase the mutual information by entangling the two qubits, i.e. is $C_{2}>C_{1}$ for the depolarising channel? To answer this question with "no" we will show that due to the isotropy of the depolarising channel the mutual information for orthogonal entangled states of two qubits depends only on the degree of entanglement, and in particular that it is a decreasing function of the entanglement. We anticipate that, as we will show later, the mutual information cannot be increased by an alphabet of non-orthogonal states.

To simplify our analysis we express the signal states in the "Bloch vector" representation 10]

$$
\pi=\frac{1}{4}\left\{\mathbb{1} \otimes \mathbb{1}+\mathbb{1} \otimes \sum_{k} \lambda_{k}^{(2)} \sigma_{k}+\sum_{k} \lambda_{k}^{(1)} \sigma_{k} \otimes \mathbb{1}+\sum_{k l} \chi_{k l} \sigma_{k} \otimes \sigma_{l}\right\}
$$

where as before $\sigma_{k}$ are the Pauli operators of the two qubits. Using Eq. (1) the output of the channel can be written as

$$
\rho=\Phi(\pi)=\frac{1}{4}\left\{\mathbb{1} \otimes \mathbb{1}+\eta \sum_{k}\left(\mathbb{1} \otimes \lambda_{k}^{(2)} \sigma_{k}+\lambda_{k}^{(1)} \sigma_{k} \otimes \mathbb{1}\right)+\eta^{2} \sum_{k l} \chi_{k l} \sigma_{k} \otimes \sigma_{l}\right\} .
$$

This shows that the output states are linked to the input signals by an isotropic shrink of the Bloch vectors $\lambda_{k}^{(1)}, \lambda_{k}^{(2)}$ by a factor $\eta$ and of the tensor $\chi_{k l}$ by a factor $\eta^{2}$.

We will now consider the following general signal state

$$
\left|\pi_{i}\right\rangle=\cos \vartheta_{i}|00\rangle_{i}+\sin \vartheta_{i}|11\rangle_{i} .
$$

In the above equation $\vartheta_{i} \in[0, \pi / 4]$ parametrises the degree of entanglement between the two qubits that carry the signal, which we will measure with the usual entropy of entanglement $S(\tau)=-\operatorname{tr}\{\tau \log \tau\}$ [11], where $\tau=\operatorname{tr}_{1} \pi_{i}=\operatorname{tr}_{2} \pi_{i}$ denotes the reduced density operator of one qubit (note that $\tau$ is the same for both qubits as the trace can be taken indifferently over any of the two ). For the state (11) we have $S_{i}=-\cos ^{2} \vartheta_{i} \log \cos ^{2} \vartheta_{i}-\sin ^{2} \vartheta_{i} \log \sin ^{2} \vartheta_{i}$.

We point out that this is the most general choice for a signal state. This can be easily verified observing that any pure state $|\Psi\rangle$ of two qubits can be decomposed in the Schmidt basis as follows [12]

$$
|\Psi\rangle=c_{1}\left|u_{1}\right\rangle\left|v_{1}\right\rangle+c_{2}\left|u_{2}\right\rangle\left|v_{2}\right\rangle,
$$

where $c_{i}$ are real and $c_{1}^{2}+c_{2}^{2}=1$, while $\left\{\left|u_{i}\right\rangle\right\}$ and $\left\{\left|v_{i}\right\rangle\right\}$ represent orthogonal bases for the first and the second qubit, respectively. These two bases will not in general have the same orientation in the Bloch vector representation, but for our purpose this is not an impediment because of the isotropy of the depolarising channel. In other words, since there is no privileged direction for the choice of basis $\{|0\rangle,|1\rangle\}$ - they do not even have to be the same for each of the two qubits - we are free to let them coincide with the two respective Schmidt bases.

After the action of the depolarising channel, the density operator corresponding to the input state (11) takes the form

$$
\rho_{i}=\frac{1}{4}\left\{\mathbb{1} \otimes \mathbb{1}+\eta \cos 2 \vartheta_{i}\left(\mathbb{1} \otimes \sigma_{z}+\sigma_{z} \otimes \mathbb{1}\right)+\eta^{2}\left(\sigma_{z} \otimes \sigma_{z}+\sin 2 \vartheta_{i}\left(\sigma_{x} \otimes \sigma_{x}-\sigma_{y} \otimes \sigma_{y}\right)\right)\right\} .
$$

In the basis $\{|00\rangle,|01\rangle,|10\rangle,|11\rangle\}$ the output $\rho_{i}$ reads

$$
\rho_{i}=\frac{1}{4}\left(\begin{array}{cccc}
1+2 \eta \cos 2 \vartheta_{i}+\eta^{2} & 0 & 0 & 2 \eta^{2} \sin 2 \vartheta_{i} \\
0 & 1-\eta^{2} & 0 & 0 \\
0 & 0 & 1-\eta^{2} & 0 \\
2 \eta^{2} \sin 2 \vartheta_{i} & 0 & 0 & 1-2 \eta \cos 2 \vartheta_{i}+\eta^{2}
\end{array}\right)
$$


The corresponding eigenvalues are

$$
\begin{aligned}
& \alpha_{1}=\alpha_{2}=\frac{1}{4}\left(1-\eta^{2}\right) \\
& \alpha_{3,4}\left(\vartheta_{i}\right)=\frac{1}{4}\left(1+\eta^{2} \pm 2 \eta \sqrt{\cos ^{2} 2 \vartheta_{i}+\eta^{2} \sin ^{2} 2 \vartheta_{i}}\right) .
\end{aligned}
$$

They depend on the degree of entanglement, but are independent of the choice of basis.

For maximisation of the second term of the mutual information $I_{2}$, given in Eq. (6), it is sufficient to optimise independently terms of the form

$$
S\left(\rho_{i}\right)=\sum_{j=1}^{4} \alpha_{j}\left(\vartheta_{i}\right) \log \alpha_{j}\left(\vartheta_{i}\right) .
$$

Remember that the a priori probabilities add to one, so neither the number of input states nor their probabilities enter in the maximisation of the second term. As we will show later, the first term in (6) and therefore the mutual information is maximised for a set of orthogonal and equally probable states.

We can search the extremum of (16) analytically leading to the requirement

$$
\cos 2 \vartheta_{i} \sin 2 \vartheta_{i} \cdot\left\{\log \left(1+\eta^{2}+2 \eta \sqrt{\cos ^{2} 2 \vartheta_{i}+\eta^{2} \sin ^{2} 2 \vartheta_{i}}\right)-\log \left(1+\eta^{2}-2 \eta \sqrt{\cos ^{2} 2 \vartheta_{i}+\eta^{2} \sin ^{2} 2 \vartheta_{i}}\right)\right\}=0
$$

For $\eta \neq 0$ there are two solutions, namely

$$
\vartheta_{i}=0,
$$

which corresponds to non-entangled input states and turns out to be the maximum, and

$$
\vartheta_{i}=\pi / 4,
$$

which means maximal entanglement of the inputs and corresponds to the minimum of $I_{2}$.

The explicit expression for the maximal mutual information corresponding to four equiprobable orthogonal non-entangled input states is given by

$$
I_{2}^{\max }=(1+\eta) \log (1+\eta)+(1-\eta) \log (1-\eta) .
$$

Notice that this is equivalent to twice the one-shot capacity. So we have shown that in this case entanglement does not help to increase the mutual information [13].

As promised, we now justify our choice of orthogonal states by proving that the mutual information cannot be increased by using non-orthogonal alphabets. We were able to maximise the two parts of the mutual information, defined in equation (6), independently of each other for the following reasons. Note first that $S(\rho)$ is maximal for $\rho=\frac{1}{4} \mathbb{1}_{1} \otimes \mathbb{1}_{2}$. For the depolarising channel this form is achieved by any set of four orthogonal equiprobable input states. The minimum of the term $\sum_{i} p_{i} S\left(\rho_{i}\right)$ in the mutual information, however, does not depend on the orthogonality of the states, as only the eigenvalues of the output states determine the extremum. We noticed that each $S\left(\rho_{i}\right)$ can be minimised independently for each input state $\pi_{i}$. As we have shown before, $S\left(\rho_{i}\right)$ is minimal, and reaches the same minimum value, for any choice of input states of non-entangled qubits. Note that due to these reasons the same mutual information could be also reached by using a larger alphabet of non-orthogonal non-entangled states and adjusted probabilities, but it can never be improved beyond the maximum value given in (20).

As an illustration, in figure 1 we show the mutual information for the following set of equally probable orthogonal states

$$
\begin{aligned}
& \left|\pi_{1}\right\rangle=\cos \vartheta|00\rangle+\sin \vartheta|11\rangle \\
& \left|\pi_{2}\right\rangle=\sin \vartheta|00\rangle-\cos \vartheta|11\rangle \\
& \left|\pi_{3}\right\rangle=\cos \beta|01\rangle+\sin \beta|10\rangle \\
& \left|\pi_{4}\right\rangle=\sin \beta|01\rangle-\cos \beta|10\rangle
\end{aligned}
$$


as a function of $\vartheta$ and $\beta$ for $\eta=0.8$. As we can see, the mutual information is a decreasing function of the degree of entanglement. In figure 2 we report the mutual information as a function of $\eta$ for uncorrelated states and maximally entangled states. As proved above, we can see that uncorrelated signals lead to a higher mutual information for any channel with $0<\eta<1$.

To summarise, we have asked whether classical communication through a depolarising channel can be improved by entangling two uses of the channel. Our analytical results show that this is not the case: the mutual information is maximised when using orthogonal equiprobable non-entangled states. The generalisation to more than two qubits remains an open problem 14 .

\section{Acknowledgements}

We would like to thank C.H. Bennett, C. Fuchs, R. Jozsa, G. Mahler and J. Schlienz for helpful discussions. In particular, we thank A. Peres for constructive critics. This work was supported in part by the European TMR Research Network ERB 4061PL95-1412 "The physics of quantum information", by Ministero dell'Università e della Ricerca Scientifica e Tecnologica under the project "Amplificazione e rivelazione di radiazione quantistica" and by Deutsche Forschungsgemeinschaft under grant SFB 407. Part of this work was completed during the 1998 workshops on quantum information organised by ISI Foundation - Elsag-Bailey and by the Benasque Center for Physics.

\section{References}

[1] C.H. Bennett, G. Brassard, C. Crépeau, R. Jozsa, A. Peres and W. Wootters, Phys. Rev. Lett. 70, 1895 (1993).

[2] A.K. Ekert, Phys. Rev. Lett. 67, 66 (1991).

[3] R. Jozsa, in "Geometric Issues in the Foundations of Science", Eds. S. Hugett, L. Mason, K.P. Tod, S.T. Tsou and N.M.J. Woodhouse, Oxford University Press 1997, (preprint quant-ph/9707034).

[4] J. Preskill, Proc. R. Soc. London 454, 385 (1998).

[5] C.H. Bennett, C. Fuchs and J. Smolin, in Quantum Communication, Computing and Measurement, eds. O. Hirota, A.S. Holevo and C.M. Caves (Plenum Press, NY, 1997), p.79 (preprint quant-ph/9611006).

[6] K. Kraus, States, Effects, and Operations: Fundamental Notions of Quantum Theory (Springer, Berlin, 1983).

[7] B. Schumacher and M.D. Westmoreland, Phys. Rev. A 56, 131 (1997).

[8] A.S. Holevo, IEEE Trans. Inf. Theory (preprint quant-ph/9611023).

[9] C.H. Bennett, D.P. DiVincenzo and J. Smolin, Phys. Rev. Lett. 78, 3217 (1997); C.H. Bennett et al., quant-ph/9904023.

[10] J. Schlienz and G. Mahler Phys. Rev. A 52, 4396 (1995).

[11] For a recent review see M.B. Plenio and V. Vedral, Contemp. Phys. 39, 431 (1998).

[12] A. Peres, Quantum Theory: Concepts and Methods (Kluwer Academic, Dordrecht, 1993).

[13] It was pointed out that entanglement is useful in super-dense coding (C.H. Bennett and S.J. Wiesner, Phys. Rev. Lett. 69, 2881 (1992)). Notice that our scenario is different from super-dense coding because in our case both qubits are transmitted through the noisy channel.

[14] Notice that our line of argument is based on the Schmidt decomposition and therefore it cannot be directly generalised to more than two uses of the channel. 


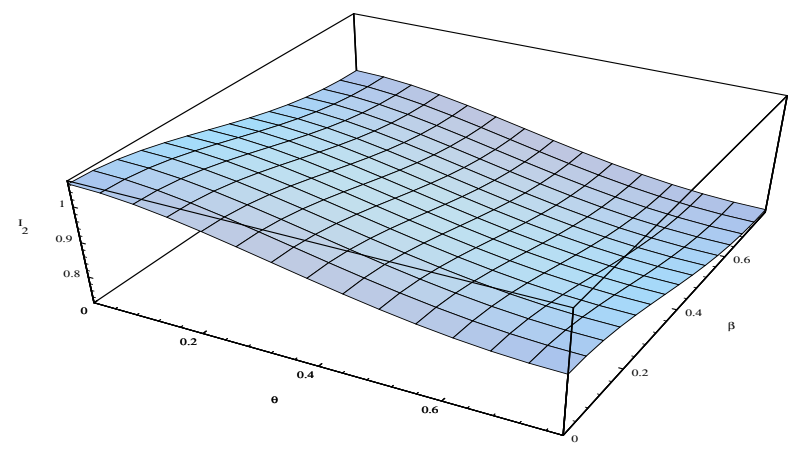

Figure 1: Mutual information for two uses of the depolarising channel as a function of $\vartheta$ and $\beta$ for $\eta=0.8$.

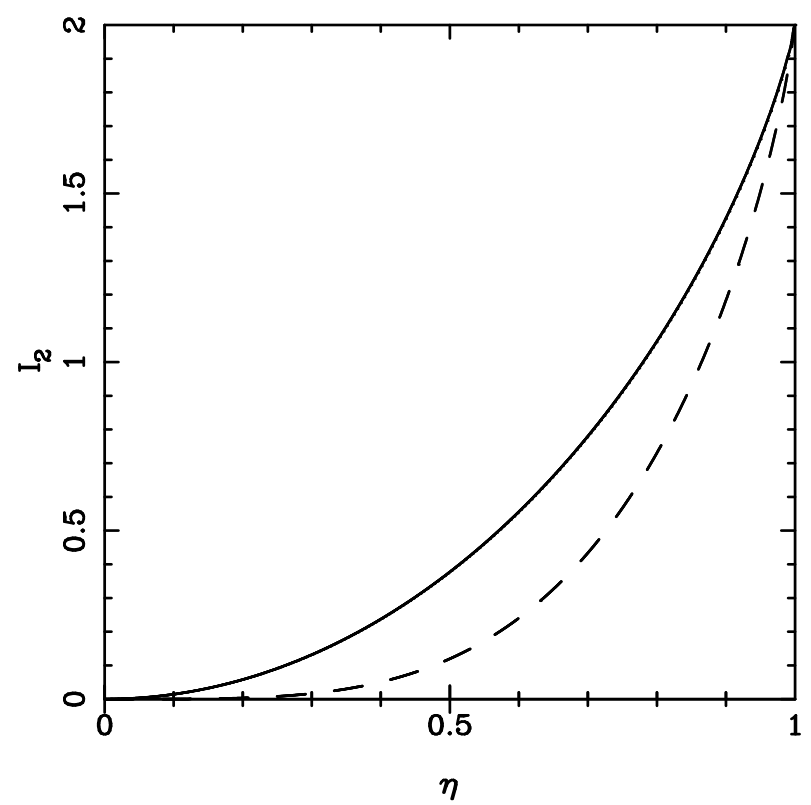

Figure 2: Mutual information for two uses of the depolarising channel as a function of $\eta$ for product input states (full line) and maximally entangled input states (dashed line). 